\title{
Les vacances de Romain Gary dans le Paris de Mai 68
}

Ce que Mai 68 a fait à la littérature, Villeneuve-d'Ascq, Presses universitaires du Septentrion, «Perspectives », p. 29-42

Mai 68 fut un virus idéaliste particulièrement contagieux. S'il n'a pas engendré chez tous les mêmes symptômes, il s'est implanté en l'homme justement parce qu'il y trouvait un biotope particulièrement favorable. Mais à l'heure où le discours sur Mai 68 est devenu surtout un contre discours, récupéré désormais par les politiques, il faut se demander plus que jamais si une thérapie radicale est véritablement souhaitable. S'il convient de vivre avec le virus ou de l'éliminer. En cela, face à cet héritage problématique, la littérature, souvent mise à l'écart des débats sur Mai 68, a peut-être son mot à dire. Sans croire qu'elle pourrait relancer la contagion ou servir d'antidote, il semble que la considérer face à 68 puisse nous renseigner un peu mieux sur ce qui s'est joué dans cet instant de bascule.

L'œuvre de Gary n'est peut-être pas la mieux placée pour nous en dire quelque chose. Il y a cela deux raisons principales. La première est d'ordre biographique. En disponibilité au ministère des Affaires étrangères depuis 1961, le romancier vit aux États-Unis au moment des événements. Il a accepté un peu plus tôt, en avril 1967, un poste de conseiller pour la diffusion de la culture au ministère de l'Information qui le contraint à une certaine réserve ${ }^{1}$. Fidèle à De Gaulle, il n'a pas pu passer du gaullisme de la France libre au «gaullisme politique $^{2} »$, et n'est jamais devenu un écrivain engagé au sens que Sartre donne à ce terme. Se proclamant, malgré cela, «gaulliste inconditionnel ${ }^{3}$ » en juin 1968, Gary affiche une dissidence qui, comme il s'en amuse, l'a souvent fait rangé parmi les réactionnaires de droite ou d'extrême-droite ${ }^{4}$.

La seconde raison est littéraire. Gary ne consacre tout simplement aucun roman à Mai 68. Alors que ses textes sont nourris de l'actualité la plus brûlante, s'adossent à l'Histoire en marche, on ne peut qu'être surpris par l'absence de cette «crise de civilisation » telle que la diagnostiquait Malraux sur l'ensemble du globe ${ }^{5}$. L'événement existe cependant dans

\footnotetext{
${ }^{1}$ Voir à ce sujet Myriam Anissimov, Romain Gary, le caméléon, Paris, Gallimard, «Folio », 2006 [2004], et David Bellos, Romain Gary: A Tall Story, London, Random House UK, 2010.

${ }^{2}$ Romain Gary, La nuit sera calme, Paris, Gallimard, « Folio », 1991 [1974], p. 232.

${ }^{3}$ Romain Gary, «Gaulliste inconditionnel », Le Monde, ${ }^{\circ} 7291,23-24$ juin 1968, p. 6

${ }^{4}$ Pour une analyse de la solidarité de Gary avec De Gaulle, aussi bien en 58 qu'en 68, voir Kerwin Spire, « Romain Gary, franc-tireur du gaullisme? », Littératures, «Les irréguliers, un autre après-guerre », Julien Roumette (dir.), $\mathrm{n}^{\circ}$ 70, 2014, p. 65-77.

5 André Malraux, Le Miroir des limbes, II, La Corde et les souris, Paris, Gallimard, « Folio », 1996 [1976]. Malraux discerne dans les utopies de 68 une défaite des Lumières et une aliénation paradoxale aux pulsions.
} 
l'univers de Gary mais il prend la forme d'un corps étranger, une quarantaine de pages glissées dans la troisième partie de Chien blanc ${ }^{6}$. Le texte, d'allure autobiographique, mettant en scène Gary dans sa résidence à Los Angeles, est une analyse sans concession de la société américaine autour des mouvements de libération noirs américains et de leurs dérives, en particulier avec les Black Panthers.

Mai 68 est donc pour part hors-jeu chez Gary. Mais cela rend-il les pages qu'il lui consacre accessoires ? C'est tout le contraire. Car ce sont précisément ces décalages et dérapages qui importent dans la lecture et le traitement de Mai 68 que propose Chien blanc. Contrairement à Malraux, Gary refuse d'aligner le printemps français sur la vague de protestation qui a secoué la planète et l'Amérique. Il le maintient sous la forme d'une digression ou d'une excroissance de son récit pour mieux nous inviter à comparer l'événement aux autres, à les différencier finement et, très certainement, à en atténuer l'éclat et le prestige. L'approche critique de Gary ose la désinvolture d'un romancier qui prend ses vacances dans le Paris de Mai sans jamais céder à une dénonciation hâtive ou partisane. L'excursus soixante-huitard, construit autour d'une série d'apologues, est chronique amusée d'une révolution condamnée à l'échec.

\section{Une révolution du spectacle}

Si l'on veut approcher la manière singulière dont Chien blanc relate Mai 68, il faut prêter garde à sa première apparition dans l'intrigue, au chapitre XIX, en sept brèves pages ${ }^{7}$, alors que Gary est encore aux États-Unis. Mai 68 vient à lui et à nous d'une manière qui est tout sauf anodine : par la télévision. Instantanément enserrée dans l'espace restreint de l'écran, l'Histoire devient à la fois information et spectacle. Non pas au sens de ce qui donne forme à l'esprit, encore moins sens, mais en tant que contenu factuel brut qui tire les choses vers le divertissement. Les actualités qui défilent ne sont ni exaltantes ni terribles : elles sont distrayantes. Pour quelle raison ? Parce que Gary les contemple depuis les États-Unis, entouré de militants noirs, de fanatisme et d'intolérance. La légèreté des manifestations parisiennes provient de la gravité de la situation américaine. Le coup de tonnerre de Mai 68 est réduit à un

\footnotetext{
${ }^{6}$ Sur ce roman, on pourra consulter Julien Roumette, « Gary et Guilloux francs-tireurs des années soixante-dix », Littératures, op. cit., p. 103-118.

${ }^{7}$ Romain Gary, Chien blanc, Paris, Gallimard, «Folio », 1983 [1970], p. 162-168. Les références à ce texte seront données entre parenthèses.
} 
bruit de grenade. Car il ne peut pas être dissocié de ce qui l'a engendré : une société du spectacle et de l'image ${ }^{8}$.

C'est donc la représentation épique de Mai 68 qui sera la surface à écailler, à traverser, alors même que Paris s'offre, en apparence, sans fard à Gary fraîchement débarqué des ÉtatsUnis : «Je trouve Paris toutes tripes dehors, débordant de détritus dans un immense élan de sincérité. On dirait que la Révolution fait passer la ville aux aveux » (173). Éventrée, la ville ne livre pourtant pas ses secrets d'elle-même. Il va falloir l'arpenter et la forcer à lever le voile sur les dessous de cette « Révolution».

Les apparences donc, comme premier point de contact, premier lieu de dénudement. Et parmi elles, les plus accessibles d'abord: les modalités d'expression de la Révolution. Car Mai 68 fut une révolte particulièrement bruyante et bavarde ${ }^{9}$. La parole s'y déchaîne. Gary note qu'elle se déploie au sein d'une forme privilégiée et rigide qui est le slogan, aussi bien pour les chansons entonnées dans la rue («Les bourgeois, c'est comme les cochons. / Plus ça devient gras, plus ça devient con! (175)) que pour les inscriptions sur les murs («Empaillez de Gaulle ! / C.R.S., S.S. ! / Le fascisme ne passera pas ! » (174)). Le romancier n'y décèle aucune manifestation spontanée venue d'une rue qu'on pourrait concevoir comme lieu de rassemblement, d'expression et de communication. Il ne déchiffre pas ces formules en tant que signes de l'émergence d'une liberté de pensée et d'une nouvelle communauté. Car ces préceptes, frappants et concis, sous forme d'assertions et d'injonctions, visent avant tout à exprimer une idée simple, voire simpliste, à être saisis dans l'instant, à valoir comme signes de ralliement et de reconnaissance. Ils sont le contraire d'une pensée autonome et singulière : basés sur des patrons syntaxiques identiques et des motifs ressassés, ils sont l'expression d'un conformisme moutonnier et d'une révolte imitée. Aussi Gary en conclut-il que «les milliers de graffiti qui couvrent les murs de Paris, tous ces slogans griffonnés sur les affiches consacrent la victoire de la publicité de marque » (174). À contre-courant, Gary ne salue pas Mai 68 comme une grande fête anticapitaliste mais y discerne, précocement et avec acuité, les séquelles de la société de consommation jusque dans ses modes d'expression.

Une première saynète vient illustrer cette réflexion. Gary est tombé sur un ancien camarade, B., en train de méditer devant l'une de ces inscriptions. B. dégaine un carnet et y consigne d'autres formules calquées sur le même modèle : «Libérez Thaelmann ! / Franco,

\footnotetext{
${ }^{8}$ Pour une étude des différentes interprétations de Mai 68 et des mythes qui lui sont associés, on renverra entre autres à Michelle Zancarini-Fournel, Le Moment 1968, une histoire contestée, Paris, Seuil, «L'Univers historique », 2008.

${ }^{9}$ Voir par exemple Maurice Tournier, Les Mots de Mai 68, Toulouse, Presses Universitaires du Mirail-Toulouse, 2007.
} 
no pasarán! Chiappe, au poteau ! / À bas la Cagoule! (174). Gary nous propulse en arrière dans le temps : nous quittons Mai 68 pour rejoindre les années 30 avec leur situation politique troublée où s'affrontent communisme et fascisme. Le romancier se prête alors très volontiers au jeu de son compagnon ; l'un et l'autre se lancent dans une surenchère de prescriptions :

\author{
«Libérez Dimitrov! Vengeons Matteotti! Sauvez l'Éthiopie! (174) \\ «La Roque ne passera pas! / J. P. assassins ! / Désarmez les ligues ! / Ils ont tué Roger \\ Salengro!»(174-175) \\ «Libérez Carl von Ossietzky! / À bas les deux cents familles ! Vengeons Guernica!
} Tous à Teruel! » (175).

Les deux camarades se créent un petit Mai 68 rien que pour eux et bien à eux, situé dans un passé politique agité où la menace fasciste et xénophobe veille. Les luttes soixantehuitardes perdent peu à peu de leur grandeur et de leur spécificité face à ce déferlement mêlant combats de premier plan et déclarations burlesques à l'emporte-pièce. Un tel pingpong linguistique n'est pas sans conséquence sur le combat de Mai 68 qui se trouve réduit à des manifestations verbales préfabriquées et stéréotypées. L'énumération en dialogue ne se clôt d'ailleurs que de façon arbitraire: d'une manière ou d'une autre, elle aurait pu se continuer presque infiniment, en variant seulement les objets, les sujets et les époques. La productivité inépuisable du slogan tend à la recette : cette facilité déconcertante à engranger des formules presque homologues et interchangeables, s'étirant au-delà de ce qui singularise des revendications et des périodes, tend à la gratuité. Mais la dépense verbale est tout sauf désintéressée : elle a fait naître le sentiment revigorant de participer à l'Histoire. « Nous nous serrons encore une fois la main avec émotion, note Gary, et je le quitte, soulagé, je marche fièrement, la tête haute. J'ai pris part à la lutte, moi aussi. » (175) Le militantisme de Mai pourrait n'être qu'un engagement du verbe devenu l'ersatz le plus commode pour remplacer actions et hauts-faits.

\title{
Le carnaval de la provocation
}

Simulacre d'engagement donc, étant donné que Gary associe ces prises de position en faveur de Mai aux classes privilégiées. Qu'y trouvent-elles? Le moyen efficace de se procurer un vertige, celui de se mettre au service des défavorisés mais sans renoncer à leur confort matériel. Un deuxième épisode illustratif pose les bases de cette observation : 
« J'hésite entre une choucroute chez Lipp et un cassoulet chez René, mais, depuis qu'il y a la Révolution, les restaurants sont bondés. Je me case tout de même chez Lipp, où une jeune fille, à la table voisine, explique que les C.R.S. ont déjà tué une centaine d'étudiants et ont jeté les corps dans la Seine, pour que ça ne se sache pas. Elle est en train de se taper un bœuf gros sel, tout en donnant des détails précis sur les étudiantes violées dans les commissariats, où l'on achève aussi les blessés. Elle commande un mille-feuille. J'ai une envie terrible de ce mille-feuille, mais ça fait engraisser. La jeune femme finit le gâteau à la hâte et se lève, avec ses amis : ils vont finir la nuit sur les barricades. Je demande à Roger Cazes comment va le ravitaillement:

- On tient, me dit-il. » (175)

Gary ne s'excepte nullement de la critique, sans quoi elle n'aurait aucune portée. Il ne cherche absolument pas, comme Sartre, à rédimer le fossé qui sépare sa condition de bourgeois avec son statut d'intellectuel progressiste. La chose est d'autant plus aisée que Gary sait que son gaullisme lui interdit de parler au nom du peuple. «Je suis gaulliste, persiflait-il avant d'arriver à Paris. Politiquement, je frise donc l'extrême droite » (170). Gary a l'esprit tranquille puisqu'il n'attend rien pour lui-même de Mai 68. Il sait qu'il est un étranger à cette révolution et peut y promener le regard étonné des Persans de Montesquieu. Il y pénètre avec un détachement réel et sincère, sans jouer au bourgeois devenu militant. Tel est le point de départ d'une inversion que Gary va progressivement assumer dans la suite. Il va mettre en place un carnaval en quelque sorte lui-même à l'envers puisqu'il n'y s'agira pas de renverser le haut par le bas mais d'exhiber l'appartenance à la bourgeoisie dans un moment où celle-ci est stigmatisée par la révolution afin d'en faire un révélateur des hypocrisies idéologiques de Mai.

Cette transformation, qui est une protestation contre les faux-semblants de ceux qui veulent mettre à bas la bourgeoisie comme concept sans y renoncer en tant que mode de vie, est déclenchée par un événement. Alors que Gary se promène accompagné par un Noir, Kaba, un C.R.S., « rubicond, du genre Roi Pausole casqué, qui vous sent le terroir, le bon vin et le bon foutre, caparaçonné jusqu'aux bottes, le bouclier de saint Louis à la main »(191), l'empêche de s'avancer et le frappe :

«Je prends un coup de matraque sur la nuque : un moment d'indignation, et puis, brusquement, tout s'éclaire : j'ai une barbe, je porte un blue-jean, un blouson, pas de cravate et, comble de mal habillé, je suis en compagnie d'un jeune Noir. Ce coup de matraque ne me vise pas personnellement. Il est purement vestimentaire. Je suis mis en salopard. Le Roi Pausole s'est trompé de classe.

Des larmes de gratitude me montent aux yeux. Foi de bourgeois, je suis défendu. Ce n'est pas pour rien que je paie des impôts. Ce coup de matraque que j'ai pris sur la gueule prouve que je suis protégé contre la canaille. Je ressens une merveilleuse sensation de sécurité. » (192)

Gary peut donc célébrer l'efficacité de la répression qui défend impulsivement le bastion bourgeois à partir de ce en quoi celui-ci croit le plus : les apparences. La bourgeoisie 
est un monde de signes et le C.R.S. y souscrit en les reconnaissant sans coup férir. Il y a pourtant comme une légère dissonance qui se fait entendre: le C.R.S. «ressemble énormément à Sa Majesté le Roi Carnaval de ma chère ville de Nice presque natale » (191). Le signe carnavalesque est pour Gary un signal ou une autorisation à carnavaliser le signe bourgeois. Car la dramaturgie de Mai ne connaît pour l'heure que deux registres : la tragédie bourgeoise qui se voue corps et âme à la répression et la comédie bourgeoise qui s'adonne hypocritement à la révolution. Gary sait que seul le carnaval pourra faire se rejoindre ces deux options qui n'en sont, au fond, qu'une.

La posture du romancier est délibérément hétérodoxe, couplant l'embourgeoisement manifeste de l'écrivain-touriste au refus d'être mis sur un pied d'égalité avec le commun du peuple. Gary revêt «tous les signes extérieurs de l'ordre bourgeois » pour s'afficher en « gaulliste notoire» (198). Il se pourvoit d'attributs sociaux forts et explicites : son « costume pied-de-poule le plus distingué » (193), sa « rosette de la Légion d'honneur », son « Homburg hat des grandes occasions, fait sur mesure chez Gellot» et «le parapluie » car « on ne peut pas sans ça ». Il lance à Kaba, le Noir qui l'accompagne : «vous allez me laisser. Vous allez mal avec ma tenue. Allez, filez. Je vais faire la Révolution. » Gary efface tout entier son statut d'homme de lettres et de sympathisant des opprimés derrière ce costume de professionnel du gaullisme et de l'ordre bourgeois, absolument impensable pour un révolutionnaire.

Encore faut-il savoir de quelle révolution on parle. Car tout porte à penser que le mot « révolution » n'a peut-être pas le même sens dans la bouche de Gary costumé en gaulliste que dans celle des manifestants. Deux révolutions pourraient bien s'affronter désormais : celle qui se déroule dans la rue, et celle que Gary cherche à déclencher. Mais plus que de révolution, il faudrait parler de protestation ou de « provocation » (193), destinée à « verser de l'huile sur le feu de la colère »(193). En effet, le petit défilé de Gary paré des signes extérieurs de la bourgeoisie s'efforce d'accroître le scandale de la richesse dans un monde en crise. Gary surjoue pour cela les codes des classes supérieures : «Je sors mon mouchoir de soie de chez Hermès et je m'essuie les yeux. Les gaz. Je m'aventure sur le boulevard SaintMichel, toutes décorations dehors. Les étudiants s'écartent en se bouchant le nez. » (195) On ne sait si les manifestants se détournent indisposés par les gaz ou par la présence de ce gaulliste répugnant. D’autant que, «tiré à quatre épingles » (193), Gary est désormais arrêté «poliment» par les C.R.S. qui reconnaissent en lui un de ceux qu'ils sont chargés de protéger.

Mais l'ordre bourgeois n'est pas seulement maintenu par la police et le gouvernement : règne aussi en sous-main chez les manifestants. Les intérêts personnels n'ont 
pas été muselés par les idéaux révolutionnaires, comme le démontre cette conversation entre un «père bourgeois cossu et son fils trotskiste discutant ensemble de la meilleure façon de faire prospérer le petit magot révolutionnaire » (197) : «c'est le triomphe de la logique sur les idées... » Autre preuve, sous forme de fable à nouveau : une jeune femme s'avance vers Gary exhibant fièrement ses signes de gaullisme pour lui demander de porter secours aux ouvriers de Renault. Gary, un instant, est ému par cet appel et cette pureté. Mais l'histoire tourne court et chute quand la jeune femme finit par réclamer à Gary, comme le font les Black Panthers avec Jean Seberg, «quelques millions » (198) : «Je regarde cette femme pour la dernière fois : j'ai l'impression d'être debout dans une rue de Moscou, en 1905. Il n'en reste plus une, en Russie. La Révolution a triomphé sur toute la ligne.»(198-199) Le capitalisme et le pragmatisme l'ont emporté.

Le lent crescendo dans la provocation auquel procède Gary est ainsi destiné à dénuder pas à pas les implicites de la révolution de mai. Il aboutit à « la plus belle expérience du Paris révolutionnaire » de Gary, « dans la cour de la Sorbonne » où il se rend avec ses « décorations bourgeoises » et sa «tenue de salonnard », par ce même «goût de provocation terroriste qui anime ceux qui [lui] lancent des quolibets. »(195) Il retourne donc contre les autres leur propre attitude, se transformant en un miroir grossissant de leurs travers. Les étudiants s'en prennent alors à lui, en raison de ses liens avec Malraux, au ministère de l'Information, et celui-ci leur rétorque avec aplomb que «la culpabilité de Malraux est évidente. Dès 1936, il invente Che Guevara, Tchen, le premier "garde rouge" et Régis Debray dans ses romans, et dès 1960 il met en place ces maisons de la culture d'où est partie "la contestation". » (195) À qui sait l'entendre, la réplique de Gary fournit deux niveaux de signification tous deux pris dans les griffes de l'ironie. Le premier est politique : il nous assure qu'un gaulliste, comme Malraux, et donc comme Gary, peut, à l'encontre de tous les préjugés, soutenir les causes révolutionnaires. Le deuxième est littéraire : il fustige la naïveté de ceux qui croient que la littérature est capable de pétrir le réel et de commander l'Histoire.

Mais tout ceci n'est là que préparer l'aboutissement de la parade du romancier qui va passer par un changement brutal d'énonciation. Un destinataire surgit sans aménité, non dans un discours séparé de la narration mais dans le récit même de Gary. Ce «vous » confond en une seule adresse les étudiants de mai et le lecteur :

«Tous mes arguments sont solides et les vôtres délirants, mais c'est vous qui avez raison. Pour le constater, il suffit d'ouvrir Le Figaro du 24 juillet 1968. Sous le titre : Voyage au bout de l'horreur dans les camps où les réfugiés meurent lentement de faim, vous trouverez un article effrayant de Jean-François Chauvel sur le Biafra (...) et, juste sous le texte, il y a un joli placard 
publicitaire avec photo à l'appui : Le Nouveau Port de Plaisance de Beaulieu-sur-Mer : une Réalité. » (195)

Le contraste est révoltant. Cet écart résume à lui seul le monde où Mai 68 s'enracine : «notre société de provocation » (195). Celle qui ne vit que d'afficher les signes de la division des classes, qui n'existe que du scandale de la richesse quand d'autres meurent de faim ou sous les balles. La réclame le dit explicitement : le port de plaisance de Beaulieu-sur-Mer n'est pas un fantasme. C'est « une réalité » que la presse est chargée de mettre en vitrine, une vérité beaucoup plus tangible que le Biafra pour le lecteur du Figaro.

Aux provocations répétées de cette société, qui sont des appels au viol, répond la provocation de Gary qui a choisi d'en pousser au bout la logique obscène pour tendre aux hommes un miroir dans lequel se voir sans masque. Mais le romancier a surtout mis à nu plusieurs conséquences de cette société de provocation et du défilé d'atrocités orchestré par les médias «sur les consciences non encore avilies» (177): mener «ou bien à l'avachissement et à l'indifférence, celle qui permet au journal télévisé de vous servir ses cadavres et ses horreurs, ses misères et ses famines à huit heures du soir pendant que vous êtes en train de dîner tranquillement, ou bien à l'explosion. » (177) Mai 68, c'est aussi cela : le refus de l'accoutumance à la violence qui alimente les médias, une déflagration de colère contre un monde où le Biafra est réduit à servir le pathos télévisuel sans jamais parvenir à faire le poids contre un port de plaisance à Beaulieu-sur-Mer ${ }^{10}$.

\section{Un événement relatif}

Or Chien blanc est avant tout une exploration sans équivalent dans la littérature française de la société américaine et de ce moment de bascule qu'ont été les mouvements de libération des Noirs américains, participant à leur manière aux révoltes qui essaiment sur le globe à la fin des années $60^{11}$. Mais Gary, on l'a dit, rompt partiellement le lien qui pourrait rattacher Mai 68 à ce qui sert d'impulsion et de terreau à son roman américain, comme pour

\footnotetext{
${ }^{10}$ L'article de Gary «The baiting society » (Playboy, vol. 16, $\mathrm{n}^{\circ}$ 5, mai 1969) développe la même idée mais souligne aussi que la révolution plonge ses racines dans la dynamique même de la création artistique.

${ }^{11}$ Sur ce sujet dans Chien blanc, voir entre autres Jean-Pierre Martin, "Actualité de Gary», Littératures, «Romain Gary, l'ombre de l'histoire », $\mathrm{n}^{\circ}$ 56, 2007, p. 27-36, Julien Roumette, « Gary et James Baldwin. Les salauds, les paumés et les "frères de race" dans Chien Blanc", dans Picaros et paumés Voyous, prostituées, maquereaux, vagabonds dans l'œuvre de Gary, Julien Roumette (dir.), Paris, Classiques Garnier, « La Revue des lettres modernes », 2014, p. 207-234
} 
ne pas justifier sa présence, lui conserver cet éclat d'étrangeté, d'arbitraire, presque de gratuité $^{12}$.

C'est d'abord la structure du roman qui fait tenir cette incongruité, en reléguant Mai 68 dans quelques chapitres tardifs qui arrivent de manière un peu impromptue. Ce montage n'est pas sans effet : le lecteur est invité à lire Mai 68 à l'aune de ce qui précède, à savoir cette fine méditation romanesque sur les rouages de la discrimination, les vestiges et les séquelles de la colonisation, le racisme, la «chiennerie» mimétique (125), la domination. Avant d'observer les manifestations de mai et la tranquille désinvolture de Gary, le lecteur aura assisté aux émeutes raciales qui ont suivi l'assassinat de Martin Luther King (66-75). Il aura participé à « un moment historique où l'on voit s'annoncer, aussi faiblement que ce soit, la naissance d'une civilisation nouvelle » (77). Le résultat de la comparaison est imparable : Mai 68 fait forcément pâle figure à côté. Évidemment, tout n'est pas disproportion dans cette mise en perspective. Sans faire système, des échos assurent une cohérence dans la vision du monde qui se cristallise : la société de provocation, explorée des deux côtés de l'Atlantique, ou les démons de la culpabilité, que ce soit chez les riches blancs américains, comme Jean Seberg qui paie « un impôt sur la "culpabilité” » (39), ou chez les soixante-huitards parisiens.

C'est donc tout autre chose que la parenté entre Mai 68 et les protestations internationales contre l'oppression qui motive l'insertion de l'épisode : le désir de prendre des vacances. Mais pas n'importe lesquelles. Il ne s'agit pas de partir visiter la capitale et de rencontrer inopinément Mai 68. Non, Gary est «saturé de négritude américaine » (162) et estime que sa «conscience elle aussi a droit à des vacances » (166). Le moyen est tout trouvé :

«Heureusement, ça bouge en France, et c'est pour moi comme une bouffée d'air frais. Rien de tel pour vous changer les idées. La télévision n'arrête pas. Prise de la Sorbonne, des milliers d'étudiants sur les barricades, menaces de grève générale. Je respire un peu. » (162)

«Paris, avec son printemps, avec ses barricades qui fleurissent, la chevalerie C.R.S., voilà exactement ce qu'il me faut pour me changer les idées. » (166)

Gary décide donc de «laisser tomber dix-sept millions de Noirs américains pour une petite virée dans le Paris du mois de mai » (165). Le renversement confine au comique de situation puisque Gary fuit une situation historique brûlante en en rejoignant une autre. Le

\footnotetext{
${ }^{12}$ Pour une analyse de la relation de ces mouvements américains avec Mai 68 et les intellectuels français, voir par exemple Beth Mauldin, «Searching for the Revolution in America: French Intellectuals, Black Panthers and the Spirit of May 68 », Critique - Journal of Socialist Theory, vol. 36, 2008 - Issue 2, p. 219-243.
} 
présupposé est clair : Mai 68 n'est pas un événement du même ordre, qui n’implique pas de la même manière la responsabilité individuelle.

Gary est cependant rattrapé par la question noire en plein cœur de ses vacances, apercevant un Noir qui, au milieu des émeutes, chante «burn, baby, burn» (176). Et le romancier de commenter :

\footnotetext{
«Entendre ce cri de guerre du Black Power américain à Sèvres-Babylone me donne chaud au cœur, éveille en moi une nostalgie que seuls peuvent comprendre ceux qui ont laissé làbas, très loin, à Beverly Hills, une piscine chauffée, une Oldsmobile air-conditionnée et quatorze chaînes de télévision, sans compter les chaînes "éducatives". » (176)
}

Cette nouvelle nostalgie, inversion ironique de celle qui était à l'origine du voyage de Gary à Paris, fait l'impasse totale sur le trop-plein de négritude américaine et rappelle à nouveau l'empire de la société de provocation. Malgré cette boutade, c'est l'irruption de ce Noir américain, le surgissement de ce que Gary s'est évertué à fuir, cette réalité violemment historique qui l'amènent à théoriser les raisons du sentiment de légèreté qui l'habite en plein Mai 68 :

«Et il est tout près de la vérité, cet Américain, face à la flamme française. Car, pour moi, aucun doute : lorsque nos C.R.S. se jettent en avant, matraque au poing, à Sèvres-Babylone, c'est au ghetto américain qu'ils ont affaire, au Viêt-nam, au Biafra et à tout ce qui crève de faim sur la terre. La révolte de la jeunesse de Paris s'inscrit tout naturellement dans ce récit parce qu'elle ne vise aucune situation sociale spécifique : elle les vise toutes. Ces poings français serrés, ces poings blancs, ce sont aussi des poings noirs. Il n'y a aucun doute là-dessus. Depuis la télévision et le transistor, le monde qui nous bombarde de ses indignités est devenu une immense entreprise de provocation, et vous vous attaquez à ce que vous avez sous la main, vous cassez tout, vous vous exprimez. Pompidou paie pour l'assassinat de Che Guevara. C'est ainsi que, d'une certaine façon, inattendue, les étudiants de Paris sont en train de renouer, en lui donnant cette fois un contenu authentique, avec la vénérable "tradition humanitaire française" et même avec notre "mission universelle". » (176)

Mai 68 en France est le nom d'une révolte générale et universelle contre toutes les situations d'oppression à travers le monde. Elle est l'expression d'une indignation face aux horreurs de l'Histoire et là se tient sa dignité. Sauf que Gary ajoute :

«S'il n’y avait pas la condition noire, sud-américaine, le Viêt-nam, le Biafra et la servitude ailleurs, la Révolution des étudiants de Paris ressemblerait singulièrement à une émeute de souris dans un fromage. » (177)

Mai 68 a cette singularité incroyable, et telle est assurément toute l'originalité de la pensée romanesque que Gary développe : ne prendre sens que dans ce qui n'est pas lui, dans une revendication pour l'autre sans avoir de revendication pour soi. Une émeute de souris 
dans un fromage : l'étudiant français a en effet à sa disposition tout ce dont il a besoin pour vivre. Il n'a pas de combat personnel à mener. Sa protestation ne peut lui appartenir ni viser sa situation : elle n'existe que pour d'autres. Dès 1970, Gary perçoit donc Mai 68 comme un événement relatif, non pas en ce qu'il serait inscrit dans la cascade de protestations qui chambarde le monde, mais parce que son contenu, son sens et sa portée ne résident pas en lui. C'est pourquoi cette révolution fait à Gary « l'effet d'une bleuette. » (181)

Cette impression est consolidée par le point de vue d'où Mai 68 est observé : 1'Amérique. Ballard, un Noir américain à Paris, fait écho aux déclarations de Gary : «Ces types-là n'ont pas de problème à eux. Tous leurs problèmes, ce sont toujours ceux des autres. Le Viêt-nam, le racisme chez nous, le Biafra, l'Afrique du Sud, la Tchécoslovaquie... ils sont toujours chez les autres... » (184). Et il ajoute : «Il faut les entendre parler du "problème noir", boulevard Saint-Michel. C'est comme s'ils avaient réussi leur vie. Il paraît que c'est la lutte des classes, le problème noir, le capitalisme. Ils n'ont pas la moindre idée de quoi ils parlent. Pour comprendre ça, il faut être américain. » (184) Les révolutionnaires de mai sont justifiés par les causes qu'ils embrassent et qu'ils lisent à travers une idéologie ne correspondant pas à une réalité vécue mais à une conception abstraite et théorique. Ballard n'est plus que l'incarnation d'une idée :

« la seule chose qu'ils voient en moi, ces petits Français, c'est le "problème". (...) Les États-Unis, pour eux, c'est pourri, c'est dégueulasse, c'est de la merde. (...) C'est comme si j'étais pas américain à leurs yeux, parce que j'ai la peau noire. C'est tout ce qu'ils voient en moi, la peau noire... » (186)

\section{Vacances en Mongolie extérieure}

De sorte que cette aspiration à la fraternité avec les minorités opprimées est en partie une construction idéologique plus qu'un sentiment sincèrement éprouvé. C'est ce que Gary tente de révéler en défilant dans les rues en gaulliste endimanché et en taxant de «sale Juif » (194) un manifestant qui l'insultait :

«Cette fois, j'ai visé juste. Il n’y a rien qui mette plus en rogne les travailleurs que de s'entendre traiter de "sales Juifs". Je sais exactement ce qu'ils ressentent : c'est comme lorsque je me fais traiter de "sale Français" en Amérique. Toute ma peau devient alors un drapeau tricolore. Il y a une sorte de vague humaine qui roule vers moi, j'effectue un repli stratégique vers les C.R.S. » (194)

«Je suis assez content : je sens que j'ai ranimé la flamme sacrée. Il y a sûrement parmi eux de bons petits gars bien de chez nous, alors, vous imaginez...» 
Gary raille le nationalisme larvé de ces champions de la fraternité, en mettant en lumière les haines sous-jacentes, comparant d'ailleurs ce qui n'est pas comparable : l'exemple américain est biaisé puisque les Juifs dont il s'agit sont aussi des Français, laissant deviner l'un des plus vieux crédo antisémite qui leur refuse l'appartenance nationale. Et Gary dira plus loin que, en tant que gaulliste, il est « exclu par tous les statuts en vigueur de ce "nous sommes tous des Juifs allemands" scandé par les étudiants français dans les rues de Paris. » (198) Mais il en est surtout exclu parce qu'il est conscient des limites de ce sentiment spontané et facile d'identification qui ne repose sur aucun fondement réel ${ }^{13}$.

C'est donc malgré tout par cette fraternité controversée que l'épisode de Mai 68 regagne les territoires intellectuels explorés par l'ensemble du roman. Son argument porte sur un chien blanc, Batka, dressé à l'attaque des Noirs, et sur les tentatives malheureuses de Gary pour le rééduquer. Il propose, comme Adieu Gary Cooper, une réflexion éthique sur la notion de communauté. Le sentiment central qui est à l'épreuve est celui de la fraternité (22), d'abord ressentie par Gary comme une véritable solidarité avec Batka. Une identification spontanée et tout à fait authentique se réalise avec lui, contrairement à ce qui est observé chez les manifestants de mai. Mais celle-ci ne présuppose jamais de se rallier à une majorité qui, toujours, conditionne les attitudes des uns et des autres. Et Mai 68, justement, a quelque chose de la meute qui vient confirmer les intuitions de la Critique de la raison dialectique de Sartre sur la foule et le groupe en fusion. Gary a tout ceci en horreur et constatait dans Adieu Gary Cooper, paru en 1969 :

«La révolte des jeunes bourgeois contre la bourgeoisie était condamnée au canular ou au fascisme, la seule différence entre les deux étant quelques millions de morts. Il y a quelque chose de profondément répugnant à voir un cortège d'étudiants venir les bras ouverts vers des ouvriers en grève, comme des femelles en chaleur devant les vrais mâles ${ }^{14}$. »

Plus sévère encore que dans Chien blanc, Gary sexualise le groupe en fusion et lui dénie toute possibilité de solidarité authentique. Il épingle des pulsions de soumission et de domination, et il pointe surtout du doigt le désir de s'accoupler à la foule, de s'amalgamer à elle pour y araser tout relief, toute singularité, pour s'abâtardir avec le peuple et se défaire de sa condition honnie de bourgeois. L'histoire de Lenny, dans Adieu Gary Cooper, dramatise ce besoin de fuir la meute et les conformismes moutonniers, lui qui, comme Gary dans Chien

\footnotetext{
${ }^{13}$ Sur les liens des écrivains avec cet appel à l'identification, nous nous permettons de renvoyer à notre article «"Nous sommes tous des Juifs allemands": l'universalisme juif en question », Revue des Sciences Humaines, «La France des solidarités (Mai 1968-Mai 1981)», Djemaa Maazouzi et Nelly Wolf (dir.), n 320, octobredécembre 2015, p. 129-140.

${ }^{14}$ Romain Gary, Adieu Gary Cooper, Paris, Gallimard, « Folio », 2011 [1969], p. 135.
} 
blanc, rêve sans cesse de la Mongolie extérieure et qui a décidé de quitter la société américaine, celle de la provocation et du Viêt-Nam, pour faire du ski «à deux mille mètres au-dessus du niveau de la merde ${ }^{15} \gg$. La Suisse donc, à défaut de vacances en Mongolie extérieure. «C'est par là que je voudrais me tirer, soupire Gary dans Chien blanc. C'est naturellement le mot extérieur qui me plaît. » (22-23) Parti en guerre contre la «nostalgie tribale » (133) et le «psychisme de ghetto » (94) à l'œuvre dans le militantisme des Black Panthers, Gary ne pouvait que frémir en retrouvant les séductions militantes et le terrorisme du groupe au cœur de Mai 68. Paris ne sera pas pour lui une Mongolie extérieure. Ce risque du pêle-mêle où l'individu s'anéantit, ressurgit à la clôture de l'épisode de Mai 68, au moment de rentrer aux États-Unis :

\begin{abstract}
«Un ami me téléphone pour m'annoncer qu'un dernier “carré” de Français libres va descendre cet après-midi les Champs-Élysées. Le dernier "carré", c'est quelque chose à quoi je n'ai jamais pu résister. J'ai horreur des majorités. Elles deviennent toujours menaçantes. On imagine donc mon désarroi lorsque, me présentant plein d'espoir sur les Champs-Élysées, je vois déferler des centaines de milliers d'hommes qui donnent une telle impression d'unanimité que j'en ai la chair de poule. Immédiatement, je me sens contre. Venu pour brandir le drapeau tricolore à la croix de Lorraine sous les risées en compagnie de quelques centaines d'autres irréguliers, je me sens volé. Je leur tourne le dos. Tous les déferlements démographiques, qu'ils soient de gauche ou de droite, me sont odieux.
\end{abstract}

Je suis un minoritaire-né. » (204-205)

Le scandale est sans compensation: même les Compagnons de la Libération, regroupement sacré pour Gary, préservé des déboires des cénacles et des bataillons, est rattrapé par l'esprit de régiment qui enfièvre Mai 68. L'événement ne pourra donc pas être le lieu d'un réveil des identités et des singularités parce qu'il est d'abord un mouvement de masse. Il ne peut être que le terreau de l'individualisme et non de l'individualité.

Gary a malgré cela croisé un frère pendant ses vacances en Mai 68 qui n'est autre que le Noir qui a déclenché sa prise de conscience de la nature relative de l'événement. Celui-ci est en réalité un écrivain et une complicité s'établit entre eux quand ils envisagent un roman sur « une mère de famille blanche qui s'envoie en l'air uniquement avec les Noirs, parce qu'avec les Noirs ça se passe dans un autre monde, elle n'a pas l'impression de tromper son mari », qui «va vivre avec un Noir, mais le trompe avec un Blanc, pour procurer à son amant noir un merveilleux sentiment d'égalité raciale avec les Blancs» (178). «Et l'histoire se prolonge en n'épargnant personne » (178) : Gary a décelé « une petite étincelle de complicité terroriste » chez celui qui pourrait être « un frère de race » (178). La seule communauté qui soit, la seule fraternité véritable se tiendrait là : dans cette dissidence assumée, cet humour

\footnotetext{
${ }^{15}$ Ibid., p. 108.
} 
séditieux dont la générosité même est de n'exempter personne, pas même soi-même, comme l'a bien montré le défilé de Gary sur son trente-et-un gaulliste.

Avec Gary, nous sommes donc face au cas unique d'une œuvre qui, battant en brèche tous les engagements, est restée à l'écoute de l'Histoire et de ses errements. Sa pensée romanesque est éminemment analytique. Reposant sur l'humour et le paradoxe, elle cerne avec vivacité et acuité les mécanismes sous-jacents et dissimulés au cœur des logiques sociales et historiques. Mai 68 n'y échappe pas. Gary y développe une position volontairement hétérodoxe, dans laquelle il comprend la manière dont l'événement résulte de la surexposition médiatique à la souffrance, dont il échoue en n'ayant pas de revendication personnelle à faire valoir. La défiance de Gary, on l'aura compris, vient surtout de sa répulsion pour les bannières, les banderoles, les slogans, toutes ces métonymies de la grégarité où naissent les doxa et les fanatismes. Car l'idéal tourne vite à l'idéologie, la passion est facilement récupérée par l'esprit de parti. C'est le sort qui attend les «groupements léninistes-trotskistes-révolutionnaires qui sortent partout du sol en ce moment, champignons succulents dont se délectent depuis toujours en salade les véritables connaisseurs comme Staline. » (197) Et c'est à ce titre que Mai 68 rejoint toutes les autres révolutions du passé, Gary constatant, revenu de toutes les illusions sans jamais désespérer pour autant : «Une révolution qui triomphe, c'est encore une révolution de foutue. Essayez donc de me démentir, donnez-moi donc un exemple historique du contraire. » (196) 